\title{
Timeline Followback Method
}

National Cancer Institute

\section{Source}

National Cancer Institute. Timeline Followback Method. NCI Thesaurus. Code C105552.

A calendar-based measure for assessing self-reported retrospective daily estimates of alcohol, tobacco and/or illicit substance use. 